\title{
SYNTHESIS OF THIAZOLIDINE-CARBAZOLE LINKED 1,2,3- TRIAZOLE HYBRIDS AND THEIR ANTI-CANCER EVALUATION
}

\author{
B. Ashok Kumar ${ }^{1,5}$, Sardar Hussain ${ }^{2}$, Balogi Gugulothu ${ }^{3}$, Sreenivas Enaganti ${ }^{4}$ \\ and J. V. Shanmuk Kumar ${ }^{5,}$ \\ ${ }^{1}$ Department of Chemistry, Sreenidhi Institute of Science \& Technology (SNIST), Yamnampet, \\ Ghatkesar, Hyderabad-501301, Telangana, India. \\ ${ }^{2}$ Department of Biotechnology, Govt. Science College, Chitradurga, Karnataka-577501, India \\ ${ }^{3}$ Dept. of Genetics \&Biotechnology, Osmania University, Hyderabad-500007, India. \\ ${ }^{4}$ Bioinformatics Division, AverinBiotech Pvt. Ltd. Windsor Plaza, Nallakunta, Hyderabad, \\ Telangana-500044, India. \\ ${ }^{5}$ Professor \& Head of Department of Chemistry, Koneru Lakshmaiah Education Foundation, \\ Green Fields, Vaddeswaram, Guntur (Dist.), Andhra Pradesh- 522502, India. \\ ${ }^{\square}$ Corresponding Author: mailme2ashokkumar@gmail.com
}

\begin{abstract}
Here we described the synthesis of novel 1,2,3-triazole linked thiazolidine-2,4-dione and carbazole derivatives and screened for their anticancer activities against three human cancer cell lines, MCF-7, HeLa and SKOV3 using MTT assay. The newly synthesized triazole hybrids are characterized using ${ }^{1} \mathrm{H} N M R,{ }^{13} \mathrm{C} \mathrm{NMR}$, IR and Mass spectral data. The results indicated that the most potent compound in this series is compound $3 \mathrm{~b}$ against MCF-7, compound $3 \mathrm{i}$ against $\mathrm{HeLa}$ and compound $3 \mathrm{~d}$ against SKOV3, which showed the highest activity with $\mathrm{IC}_{50}$ value of 32.92 $\mu \mathrm{g} / \mathrm{ml}, 12.08 \mu \mathrm{g} / \mathrm{ml}$ and $29.06 \mu \mathrm{g} / \mathrm{ml}$ respectively.
\end{abstract}

Keywords: 1,2,3-triazole, Carbazole, Thiazolidine-2,4-dione, Anticancer Activity

RASĀYAN J. Chem., Vol. 14, No.3, 2021

\section{INTRODUCTION}

In the recent era of medicinal chemistry, a unique pharmacophore with the combination of two lead moieties is the usual application to obtain bioactive hybrid compounds with novelty and high active that have attributed a new prospect in multifactorial disease therapeutics. In this molecular hybridization concept, the compounds obtained may be due to binding of multiple copies of the same (homo) or unique (hetero) ligands connected via linkers or a scaffold, are reported to act by inhibiting two or more conventional targets simultaneously. Regarding heterobivalent ligands, the hybrid compound is composed of two different pharmacophores and this characteristic in targeting different protein molecules or two specific sites on the same protein target. The choice of and concurrent binding of heterobivalent ligands to various targets could be beneficial for the treatment of many diseases including cancer. ${ }^{1}$ Cancer, due to which massive human deaths occur all through the world. By the year 2020, the approximation of cancer occurrence per year is 16 million new cases and more than $20 \%$ of the population is affected by cancer with increasing incidence annually.,3 Granting all this, the exploration of anticancer drugs has been remarkable research, advancing attempts for the improvement of chemicals that might be advantageous in the chemotherapy of cancer. Thiazolidinediones (TZDs), considering the governance of various physiological processes, have been accountable in the wide-ranging scientific research field. Thiazolidinediones constitutes some important drugs that includes rosiglitazone, pioglitazone, ciglitazone and troglitazone. On the other hand, glitazones have been outlined in recent times due to their peculiar anticancer effect mechanisms. ${ }^{4}$ In the past years, these compounds have been well-established with their various functioning biological activities of antimicrobial, antimalarial, anti-inflammatory, anticancer and antihyperlipidemic, antihyperglycemic, cholesterol esterase inhibitor, 15-hydroxyprostaglandin 
RASĀYAN J. Chem.

Vol. 14 | No. 3 |1551-1557| July - September | 2021

dehydrogenase(15-PGDH) inhibitorand bacterial arylamine N-Acetyltransferases (NATs) inhibitor. ${ }^{5-15}$ On the other hand, carbazoles and their derivatives attained great significance for medicinal chemists for the reason that they possess various biological activities. Carbazole is an aromatic heterocyclic organic compound found in distinct natural products and drug molecules. This carbazole framework illustrates a vast range of biological and pharmacological activities inclusive of antibacterial, anti-inflammatory, anticancer, antitubercular, antidiabetic, antioxidant, anti-HIV and inhibitors of topoisomerase II. ${ }^{16-24} \mathrm{~A}$ few carbazole derivatives are having probable multifunctional agents for the treatment of neurological disorders. ${ }^{25}$ Some of the acclaimed thiazolidine-2,4-dione and carbazole derivatives were described to be pharmacological active agents as represented in Fig.-1.<smiles>CC1(COc2ccc(CC3SC(=O)NC3=O)cc2)CCCCC1</smiles>

Ciglitazone

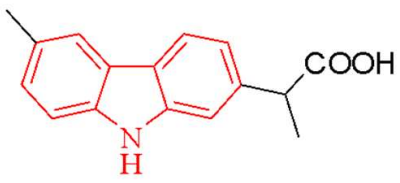

Carprofen

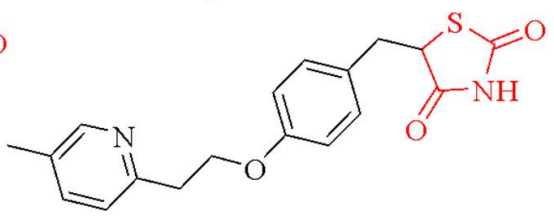

Pioglitazone<smiles>Cc1ccc2[nH]c3ccc4c(c3c2c1)C=CC(C)(C)O4</smiles>

Glycomaurin<smiles>CCN1/C(=C/C=C2/SC(=O)NC2=O)Sc2ccccc21</smiles>

Rhodanine red

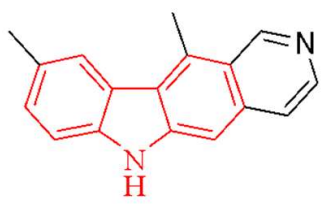

Ellipticine

Fig.-1: Some of the Selected Molecules that containingThiazolidine-2,4-dione and Carbazole Derivatives with Pharmacological Activity

\section{EXPERIMENTAL}

\section{General Information}

S.D. Fine Chemicals are used without purification. For thin-layer chromatography Merck silica gel 60F254 pre-coated plates were used and for column chromatography silica gel 60-120 mesh was used. CasiaSiamia (VMP-AM) melting point apparatus was used for reading melting points. ${ }^{1} \mathrm{H}$ and ${ }^{13} \mathrm{C}$ NMR spectra were measured on $400 \mathrm{MHz}$ and $100 \mathrm{MHz}$ spectrometers respectively, using DMSO- $\mathrm{d}_{6}$ as a solvent and TMS as a reference. Shimadzu QP5050A quadrupole-based mass spectrometer was used for recording mass spectrum.

\section{Synthesis of 5-((9-ethyl-9H-carbazol-3-yl)methylene)thiazolidine-2,4-dione (1)}

9-ethyl-9H-carbazole-3-carbaldehyde $(30 \mathrm{mmol})$ was taken in toluene and to this thiazolidine-2,4-dione (30 mmol) and piperidine was added and refluxed for four hours. After completion of the reaction as indicated by TLC the round bottom flask was cooled to $25^{\circ} \mathrm{C}$ and it was recrystallized from water to get compound 1 .

Synthesis of 5-((9-ethyl-9H-carbazol-3-yl)methylene)-3-(prop-2-yn-1-yl)thiazolidine-2,4-dione(2) 5-((9-ethyl-9H-carbazol-3-yl)methylene)thiazolidine-2,4-dione $(23.60 \mathrm{mmol})$ was dissolved in $10 \mathrm{~mL}$ of acetone solvent and $\mathrm{K}_{2} \mathrm{CO}_{3}(22 \mathrm{mmol})$ was used as base. After 10 minutes 3-bromoprop-1-yne (23 mmol) was added and the reaction mixture refluxed for 3 hours and after completion of the reaction which was known by TLC, it was extracted with ethyl acetate to get 5-((9-ethyl-9H-carbazol-3-yl)methylene)-3(prop-2-yn-1-yl)thiazolidine-2,4-dione(2). It was purified by column chromatography over silica gel (100$200 \mathrm{mesh}$ ) in $10 \%$ ethyl acetate in hexane to get compound $\mathbf{2}$ in pure state.

Mp. $180-182^{\circ} \mathrm{C}$. Yield 91\%. ${ }^{1} \mathrm{HNMR}\left(400 \mathrm{MHz}, \mathrm{CDCl}_{3}\right) \delta 8.21(\mathrm{~d}, J=1.7 \mathrm{~Hz}, 1 \mathrm{H}), 8.12(\mathrm{t}, J=3.6 \mathrm{~Hz}$, 2H), 7.59 (dd, $J=8.4,1.7 \mathrm{~Hz}, 1 \mathrm{H}), 7.55-7.51(\mathrm{~m}, 1 \mathrm{H}), 7.44(\mathrm{dd}, J=8.4,3.7 \mathrm{~Hz}, 2 \mathrm{H}), 7.33-7.29$ (m, $1 \mathrm{H}), 4.50(\mathrm{~d}, J=2.5 \mathrm{~Hz}, 2 \mathrm{H}), 4.37(\mathrm{q}, J=7.3 \mathrm{~Hz}, 2 \mathrm{H}), 2.28(\mathrm{t}, J=2.5 \mathrm{~Hz}, 1 \mathrm{H}), 1.45(\mathrm{t}, J=7.3 \mathrm{~Hz}, 3 \mathrm{H})$. 
RASĀYAN $J$. Chem.

Vol. 14 | No. 3 |1551-1557| July - September | 2021

Synthesis of 1,2,3-triazole ring linked 5-((9-ethyl-9H-carbazol-3-yl)methylene)thiazolidine-2,4-dione hybrids 3a-3k

Compound $2(1.0 \mathrm{mmol})$ and aromatic azides $(1.0 \mathrm{mmol})$ were dissolved in tetrahydrofuran solvent (10 $\mathrm{mL}$ ) and copper iodide $(10 \mathrm{~mol} \%)$ was added. The reaction was maintained for $12 \mathrm{~h}$ at room temperature. After completion of the reaction which was confirmed by TLC, the solvent was removed and extracted with DCM. The organic layers were dried using anhydrous sodium sulphate and concentrated to get the final products $\mathbf{3 a - 3 k}$.

5-\{(9-ethyl-9H-carbazol-3-yl)methylene $\}-3-\{(1-p h e n y l-1 H-1,2,3-t r i a z o l-4-y l) m e t h y l\} \quad$ thiazolidine2,4-dione (3a)

Yield $81 \%$, mp 150-152 ${ }^{\circ} \mathrm{C}$. IR spectrum, $v, \mathrm{~cm}^{-1}: 3155,2923,1733,1675,1585,1456 .{ }^{1} \mathrm{H}$ NMR (400 MHz, DMSO-d $\left.{ }_{6}\right) 8.08(\mathrm{~s}, 1 \mathrm{H}), 7.80(\mathrm{~s}, 1 \mathrm{H}), 7.69-7.61(\mathrm{~m}, 2 \mathrm{H}), 7.57(\mathrm{~s}, 1 \mathrm{H}), 7.54(\mathrm{~s}, 1 \mathrm{H}), 7.45-7.34$ $(\mathrm{m}, 2 \mathrm{H}), 7.30-7.18(\mathrm{~m}, 5 \mathrm{H}), 7.04(\mathrm{~s}, 1 \mathrm{H}), 5.08(\mathrm{~s}, 2 \mathrm{H}), 4.38(\mathrm{q}, J=7.2 \mathrm{~Hz}, 2 \mathrm{H}), 1.46(\mathrm{t}, J=7.2 \mathrm{~Hz}, 3 \mathrm{H})$. ${ }^{13} \mathrm{C}$ NMR $\left(100 \mathrm{MHz}, \mathrm{DMSO}-\mathrm{d}_{6}\right) \delta 167.1,165.2,143.0,140.5,140.1,136.1,135.1,130.7,129.9,127.8$, $126.8,123.5,122.8,121.9,121.8,121.5,120.6,119.9,119.8,116.5,110.9,109.5,37.2,36.5,13.7$. ESIMS: $\mathrm{m} / \mathrm{z} 480(\mathrm{M}+1)$ observed for $\mathrm{C}_{27} \mathrm{H}_{21} \mathrm{~N}_{5} \mathrm{O}_{2} \mathrm{~S}$.

\section{5-\{(9-ethyl-9H-carbazol-3-yl)methylene\}-3-\{1-(4-nitrophenyl)-1H-1,2,3-triazol-4- yl)methyl $\}$ thiazolidine-2,4-dione(3b) \\ Yield $83 \%$, mp 162-163 ${ }^{\circ} \mathrm{C}$. IR spectrum, $v, \mathrm{~cm}^{-1}: 3154,2923,1733,1675,1585,1456 .{ }^{1} \mathrm{H}$ NMR (400 MHz, DMSO-d 6 ) $\delta 8.09(\mathrm{~d}, \mathrm{~J}=7.4 \mathrm{~Hz}, 2 \mathrm{H}), 7.99(\mathrm{~s}, 1 \mathrm{H}), 7.93(\mathrm{~d}, \mathrm{~J}=7.4 \mathrm{~Hz}, 2 \mathrm{H}), 7.80(\mathrm{~s}, 1 \mathrm{H}), 7.57(\mathrm{~s}$, 1H), $7.53(\mathrm{~s}, 1 \mathrm{H}), 7.44-7.36(\mathrm{~m}, 2 \mathrm{H}), 7.29-7.19(\mathrm{~m}, 3 \mathrm{H}), 4.88(\mathrm{~s}, 2 \mathrm{H}), 4.38(\mathrm{q}, \mathrm{J}=7.2 \mathrm{~Hz}, 2 \mathrm{H}), 1.47(\mathrm{t}$, $\mathrm{J}=7.2 \mathrm{~Hz}, 3 \mathrm{H}) .{ }^{13} \mathrm{C}$ NMR $(100 \mathrm{MHz}$, DMSO-d 6 ) $\delta 168.1,165.9,146.1,140.6,140.1,135.6,134.9,132.7$, $127.8,126.8,1233.7,123.5,122.8,121.9,121.8,121.7,121.3,120.7,119.8,116.6,110.1,109.7,37.2$, 36.5, 13.7. ESI-MS: $\mathrm{m} / \mathrm{z} 525(\mathrm{M}+1)$ observed for $\mathrm{C}_{27} \mathrm{H}_{20} \mathrm{~N}_{6} \mathrm{O}_{4} \mathrm{~S}$.}

\section{3-\{1-(4-acetylphenyl)-1H-1,2,3-triazol-4-yl)methyl\}-5-\{(9-ethyl-9H-carbazol-3- yl)methylene $\}$ thiazolidine-2,4-dione (3c) \\ Yield $80 \%, \mathrm{mp} 155-158{ }^{\circ} \mathrm{C}$. IR spectrum, $v, \mathrm{~cm}^{-1}: 3129,2940,1725,1655,1550,1439 .{ }^{1} \mathrm{H}$ NMR (400 MHz, DMSO-d 6$) \delta 9.00(\mathrm{~s}, 1 \mathrm{H}), 8.47(\mathrm{~s}, 1 \mathrm{H}), 8.25(\mathrm{~s}, 1 \mathrm{H}), 8.18-8.08(\mathrm{~m}, 4 \mathrm{H}), 7.91-7.58(\mathrm{~m}, 4 \mathrm{H}), 7.54$ $(\mathrm{s}, 1 \mathrm{H}), 7.30(\mathrm{~s}, 1 \mathrm{H}), 5.05(\mathrm{~s}, 2 \mathrm{H}), 4.50(\mathrm{q}, 2 \mathrm{H}), 2.63(\mathrm{~s}, 3 \mathrm{H}), 1.35(\mathrm{t}, 3 \mathrm{H}) \cdot{ }^{13} \mathrm{C}$ NMR $\left(100 \mathrm{MHz}, \mathrm{DMSO}-\mathrm{d}_{6}\right)$ $\delta 196.9,167.3,165.3,143.0,140.6,140.1,139.4,136.4,134.9,132.8,130.0,127.81,126.7,123.7,123.6$, 122.9, 121.9, 120.8, 119.9, 119.7, 116.6, 110.1, 109.7, 37.2, 36.5, 26.8, 13.7. ESI-MS: m/z $524(\mathrm{M}+1)$ observed for $\mathrm{C}_{28} \mathrm{H}_{21} \mathrm{~N}_{5} \mathrm{O}_{4} \mathrm{~S}$.}

\section{3-\{(1-[3,4-dichlorophenyl]-1H-1,2,3-triazol-4-yl)methyl\}-5-\{(9-ethyl-9H-carbazol-3- yl)methylene\}thiazolidine-2,4-dione(3d) \\ Yield $82 \%$, mp 132-134 ${ }^{\circ} \mathrm{C}$. IR spectrum, $v, \mathrm{~cm}^{-1}: 3156,2920,1733,1683,1518,1488 .{ }^{1} \mathrm{H}$ NMR (400 MHz, DMSO-d $\left.\mathrm{d}_{6}\right) 8.95(\mathrm{~s}, 1 \mathrm{H}), 8.46(\mathrm{~s}, 1 \mathrm{H}), 8.34-8.20(\mathrm{~m}, 2 \mathrm{H}), 8.19-8.09(\mathrm{~m}, 1 \mathrm{H}), 8.02-7.91(\mathrm{~m}$, $1 \mathrm{H}), 7.85(\mathrm{~d}, J=8.4 \mathrm{~Hz}, 1 \mathrm{H}), 7.74-7.75(\mathrm{~m}, 1 \mathrm{H}), 7.71(\mathrm{dd}, J=16.1,8.1 \mathrm{~Hz}, 2 \mathrm{H}), 7.54(\mathrm{t}, J=7.3 \mathrm{~Hz}$, $1 \mathrm{H}), 7.30(\mathrm{t}, J=7.3 \mathrm{~Hz}, 1 \mathrm{H}), 5.03(\mathrm{~s}, 2 \mathrm{H}), 4.49(\mathrm{q}, J=6.7 \mathrm{~Hz}, 2 \mathrm{H}), 1.34(\mathrm{t}, J=6.7 \mathrm{~Hz}, 3 \mathrm{H}) .{ }^{13} \mathrm{C}$ NMR $\left(100 \mathrm{MHz}, \mathrm{DMSO}-\mathrm{d}_{6}\right) \delta 167.2,165.2,142.9,140.6,140.1,135.9,135.0,132.2,131.7,131.0,127.8$, 126.7, 123.7, 123.5, 122.8, 121.9, 121.8,121.6, 120.7, 119.9, 119.8, 116.5, 110.9, 109.7, 37.2, 36.5, 13.71 . ESI-MS: m/z $548(\mathrm{M}+1)$ observed for $\mathrm{C}_{27} \mathrm{H}_{19} \mathrm{Cl}_{2} \mathrm{~N}_{5} \mathrm{O}_{2} \mathrm{~S}$.}

\section{5-\{(9-ethyl-9H-carbazol-3-yl)methylene\}-3-\{[1-(2-ethylphenyl)-1H-1,2,3-triazol-4-} yl]methyl\}thiazolidine-2,4-dione(3e)

Yield 78\%, mp $130-132{ }^{\circ} \mathrm{C}$. IR spectrum, $v, \mathrm{~cm}^{-1}: 3155,2923,1733,1675,1585,1456 .{ }^{1} \mathrm{H}$ NMR (400 MHz, DMSO-d $\left.{ }_{6}\right) \delta 8.52(\mathrm{~s}, 1 \mathrm{H}), 8.45(\mathrm{~s}, 1 \mathrm{H}), 8.25(\mathrm{~d}, J=7.7 \mathrm{~Hz}, 1 \mathrm{H}), 8.14(\mathrm{~s}, 1 \mathrm{H}), 7.78(\mathrm{~d}, J=8.6 \mathrm{~Hz}$, $1 \mathrm{H}), 7.71(\mathrm{~d}, J=9.2 \mathrm{~Hz}, 1 \mathrm{H}), 7.68(\mathrm{~d}, J=8.2 \mathrm{~Hz}, 1 \mathrm{H}), 7.57-7.47(\mathrm{~m}, 3 \mathrm{H}), 7.46-7.34(\mathrm{~m}, 2 \mathrm{H}), 7.30(\mathrm{t}, J$ $=7.4 \mathrm{~Hz}, 1 \mathrm{H}), 5.06(\mathrm{~s}, 2 \mathrm{H}), 4.48(\mathrm{q}, J=7.1 \mathrm{~Hz}, 2 \mathrm{H}), 2.43(\mathrm{q}, J=7.4 \mathrm{~Hz}, 2 \mathrm{H}), 1.34(\mathrm{t}, J=6.9 \mathrm{~Hz}, 3 \mathrm{H})$, $1.01(\mathrm{t}, J=7.4 \mathrm{~Hz}, 3 \mathrm{H}) .{ }^{13} \mathrm{C}$ NMR $\left(100 \mathrm{MHz}, \mathrm{DMSO}-\mathrm{d}_{6}\right) \delta 167.3,165.3,140.5,140.1,139.2,135.5$, 
$134.9,130.2,129.8,127.8,126.9,126.7,126.3,125.5,123.6,123.4,123.5,122.9,121.8,120.8,119.9$, 116.6, 110.1, 109.7, 37.3, 36.5, 23.7, 14.8, 13.7. ESI-MS: $\mathrm{m} / \mathrm{z} 508(\mathrm{M}+1)$ observed for $\mathrm{C}_{29} \mathrm{H}_{25} \mathrm{~N}_{5} \mathrm{O}_{2} \mathrm{~S}$.

\section{5-\{(9-ethyl-9H-carbazol-3-yl)methylene\}-3-\{[1-(4-fluorophenyl)-1H-1,2,3-triazol-4-yl]methyl\} thiazolidine-2,4-dione (3f) \\ Yield $80 \%$, mp $125-127{ }^{\circ} \mathrm{C}$. IR spectrum, $v, \mathrm{~cm}^{-1}: 3156,2920,1733,1683,1518,1488,{ }^{1} \mathrm{H}$ NMR (400 MHz, DMSO-d $\left.{ }_{6}\right) \delta 8.07(\mathrm{~s}, 1 \mathrm{H}), 7.78(\mathrm{~s}, 1 \mathrm{H}), 7.71(\mathrm{~s}, 1 \mathrm{H}), 7.70-7.65(\mathrm{~m}, 2 \mathrm{H}), 7.52(\mathrm{~s}, 1 \mathrm{H}), 7.42-7.34$ $(\mathrm{m}, 2 \mathrm{H}), 7.29(\mathrm{dd}, J=5.1,2.4 \mathrm{~Hz}, 1 \mathrm{H}), 7.27-7.19(\mathrm{~m}, 2 \mathrm{H}), 7.15-7.09(\mathrm{~m}, 2 \mathrm{H}), 4.98(\mathrm{~s}, 2 \mathrm{H}), 4.38(\mathrm{q}, J$ $=7.2 \mathrm{~Hz}, 2 \mathrm{H}), 1.46(\mathrm{t}, J=7.2 \mathrm{~Hz}, 3 \mathrm{H})$. ESI-MS: $\mathrm{m} / \mathrm{z} 498(\mathrm{M}+1)$ observed for $\mathrm{C}_{27} \mathrm{H}_{20} \mathrm{FN}_{5} \mathrm{O}_{2} \mathrm{~S}$.}

\section{3-\{(1-[4-chlorophenyl]-1H-1,2,3-triazol-4-yl)methyl\}-5-\{(9-ethyl-9H-carbazol-3-yl)methylene\} thiazolidine-2,4-dione (3g) \\ Yield $85 \%$, mp $135-138{ }^{\circ} \mathrm{C}$. IR spectrum, $v, \mathrm{~cm}^{-1}: 3156,2920,1733,1683,1518,1488 .{ }^{1} \mathrm{H}$ NMR (400 MHz, DMSO-d 6$) \delta 8.89(\mathrm{~s}, 1 \mathrm{H}), 8.46(\mathrm{~s}, 1 \mathrm{H}), 8.26(\mathrm{~d}, J=7.5 \mathrm{~Hz}, 1 \mathrm{H}), 8.15(\mathrm{~s}, 1 \mathrm{H}), 7.89(\mathrm{~d}, J=8.3 \mathrm{~Hz}$, 2H), $7.80(\mathrm{~d}, J=8.3 \mathrm{~Hz}, 2 \mathrm{H}), 7.78-7.58(\mathrm{~m}, 3 \mathrm{H}), 7.54(\mathrm{t}, J=7.4 \mathrm{~Hz}, 1 \mathrm{H}), 7.30(\mathrm{t}, J=7.3 \mathrm{~Hz}, 1 \mathrm{H}), 5.03$ $(\mathrm{s}, 2 \mathrm{H}), 4.49(\mathrm{~d}, J=6.7 \mathrm{~Hz}, 2 \mathrm{H}), 1.34(\mathrm{t}, J=6.7 \mathrm{~Hz}, 3 \mathrm{H}) .{ }^{13} \mathrm{C}$ NMR $\left(100 \mathrm{MHz}, \mathrm{DMSO}-\mathrm{d}_{6}\right) \delta 167.2,165.2$, $142.9,140.6,140.1,136.0,135.0,130.6,129.9,127.7,126.7,123.5,122.8,121.9,121.8,121.6,120.7$, 119.9, 119.8, 116.5, 110.9, 109.6, 37.2, 36.4, 13.7. ESI-MS: m/z $514(\mathrm{M}+1)$ observed for $\mathrm{C}_{27} \mathrm{H}_{20} \mathrm{ClN}_{5} \mathrm{O}_{2} \mathrm{~S}$.}

\section{3-\{1-[(4-bromophenyl)-1H-1,2,3-triazol-4-yl]methyl\}-5-\{(9-ethyl-9H-carbazol-3-yl)methylene\} thiazolidine-2,4-dione (3h) \\ Yield $80 \%$, mp 120-122 ${ }^{\circ} \mathrm{C} .{ }^{1} \mathrm{H}$ NMR (400 MHz, DMSO-d 6 ) $\delta 8.89$ (s, 1H), $8.46(\mathrm{~s}, 1 \mathrm{H}), 8.26(\mathrm{~d}, J=7.5$ $\mathrm{Hz}, 1 \mathrm{H}), 8.15(\mathrm{~s}, 1 \mathrm{H}), 7.89(\mathrm{~d}, J=8.3 \mathrm{~Hz}, 2 \mathrm{H}), 7.84-7.76(\mathrm{~m}, 3 \mathrm{H}), 7.71(\mathrm{dd}, J=16.8,8.3 \mathrm{~Hz}, 2 \mathrm{H}), 7.54$ $(\mathrm{t}, J=7.4 \mathrm{~Hz}, 1 \mathrm{H}), 7.30(\mathrm{t}, J=7.3 \mathrm{~Hz}, 1 \mathrm{H}), 5.03(\mathrm{~s}, 2 \mathrm{H}), 4.49(\mathrm{q}, J=6.7 \mathrm{~Hz}, 2 \mathrm{H}), 1.34(\mathrm{t}, J=6.7 \mathrm{~Hz}$, $3 \mathrm{H}) .{ }^{13} \mathrm{C}$ NMR $\left(100 \mathrm{MHz}, \mathrm{DMSO}-\mathrm{d}_{6}\right) \delta 167.2,165.3,140.6,140.1,135.6,134.9,132.7,127.8,126.7$, 123.7, 123.5, 122.9, 121.9, 121.9, 121.7, 121.5, 121.3, 120.8, 119.9, 116.6, 110.1, 109.7, 37.2, 36.5, 13.7 . ESI-MS: m/z $558(\mathrm{M}+1)$ observed for $\mathrm{C}_{27} \mathrm{H}_{20} \mathrm{BrN}_{5} \mathrm{O}_{2} \mathrm{~S}$.}

\section{5-\{(9-ethyl-9H-carbazol-3-yl)methylene\}-3-\{(1-[4-methoxyphenyl]1H-1,2,3-triazol-4-yl)methyl\} thiazolidine-2,4-dione (3i)}

Yield $75 \%$, mp $130-133{ }^{\circ} \mathrm{C}$. IR spectrum, $v, \mathrm{~cm}^{-1}: 3156,2926,1736,1685,1588,1451 .{ }^{1} \mathrm{H}$ NMR (400 MHz, DMSO-d 6$) \delta 8.08(\mathrm{~s}, 1 \mathrm{H}), 7.78(\mathrm{~s}, 1 \mathrm{H}), 7.71(\mathrm{~s}, 1 \mathrm{H}), 7.64-7.59(\mathrm{~m}, 2 \mathrm{H}), 7.52(\mathrm{~s}, 1 \mathrm{H}), 7.42-7.21$ $(\mathrm{m}, 7 \mathrm{H}), 4.88(\mathrm{~s}, 2 \mathrm{H}), 4.38(\mathrm{q}, J=7.2 \mathrm{~Hz}, 2 \mathrm{H}), 3.57(\mathrm{~s}, 3 \mathrm{H}), 1.47(\mathrm{t}, J=7.2 \mathrm{~Hz}, 3 \mathrm{H}) .{ }^{13} \mathrm{C}$ NMR $(100$ MHz, DMSO-d 6 ) $\delta 167.3,165.3,156.2,143.0,140.6,140.1,139.4,136.3,134.9,130.0,127.8,126.7$, 123.7, 123.5, 122.9, 121.9, 120.8, 119.9, 119.7, 116.6, 110.1, 109.7, 55.1, 37.2, 36.5, 13.7. ESI-MS: $\mathrm{m} / \mathrm{z}$ $510(\mathrm{M}+1)$ observed for $\mathrm{C}_{27} \mathrm{H}_{23} \mathrm{~N}_{5} \mathrm{O}_{3} \mathrm{~S}$.

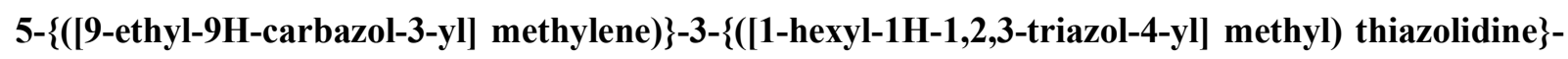
2,4-dione (3j)

Yield 73\%, mp 121-123 ${ }^{\circ} \mathrm{C} .{ }^{1} \mathrm{H}$ NMR (400 MHz, DMSO-d $) \delta 7.69(\mathrm{~s}, 1 \mathrm{H}), 7.64(\mathrm{~s}, 1 \mathrm{H}), 7.59(\mathrm{~s}, 1 \mathrm{H})$, $7.43(\mathrm{~s}, 1 \mathrm{H}), 7.32-7.28(\mathrm{~m}, 2 \mathrm{H}), 7.26-7.22(\mathrm{~m}, 1 \mathrm{H}), 7.19-7.08(\mathrm{~m}, 2 \mathrm{H}), 5.02(\mathrm{~s}, 2 \mathrm{H}), 4.38(\mathrm{q}, J=7.2$ $\mathrm{Hz}, 2 \mathrm{H}), 3.76(\mathrm{t}, J=7.2 \mathrm{~Hz}, 2 \mathrm{H}), 1.46(\mathrm{t}, J=7.2 \mathrm{~Hz}, 3 \mathrm{H}), 1.36-1.23(\mathrm{~m}, 8 \mathrm{H}), 0.88(\mathrm{t}, J=6.7 \mathrm{~Hz}, 3 \mathrm{H})$. ESI-MS: m/z $586(\mathrm{M}+1)$ observed for $\mathrm{C}_{27} \mathrm{H}_{29} \mathrm{~N}_{5} \mathrm{O}_{2} \mathrm{~S}$.

5-\{(9-ethyl-9H-carbazol-3-yl) methylene $\}-3-\{([1-o c t y l-1 H-1,2,3-$ triazol-4-yl] methyl) thiazolidine $\}-$ 2, 4-dione (3k)

Yield 72\%, mp 121-124 ${ }^{\circ} \mathrm{C}$. IR spectrum, $v, \mathrm{~cm}^{-1}: 3122,2924,1744,1684,1591,1506 .{ }^{1} \mathrm{H}$ NMR (400 MHz, DMSO-d 6$) \delta 7.62(\mathrm{~s}, 1 \mathrm{H}), 7.61(\mathrm{~s}, 1 \mathrm{H}), 7.55(\mathrm{~s}, 1 \mathrm{H}), 7.41(\mathrm{~s}, 1 \mathrm{H}), 7.30-7.26(\mathrm{~m}, 2 \mathrm{H}), 7.25-7.19$ (m, 1H), $7.16-7.01(\mathrm{~m}, 2 \mathrm{H}), 5.04(\mathrm{~s}, 2 \mathrm{H}), 4.38(\mathrm{q}, J=7.2 \mathrm{~Hz}, 2 \mathrm{H}), 3.76(\mathrm{t}, J=7.2 \mathrm{~Hz}, 2 \mathrm{H}), 1.79-1.70$ $(\mathrm{m}, 4 \mathrm{H}), 1.45(\mathrm{t}, J=7.2 \mathrm{~Hz}, 3 \mathrm{H}), 1.25-1.13(\mathrm{~m}, 8 \mathrm{H}), 0.98(\mathrm{t}, J=6.7 \mathrm{~Hz}, 3 \mathrm{H})$. ESI-MS: $\mathrm{m} / \mathrm{z} 516(\mathrm{M}+1)$ observed for $\mathrm{C}_{29} \mathrm{H}_{33} \mathrm{~N}_{5} \mathrm{O}_{2} \mathrm{~S}$. 
RASĀYAN J. Chem.

Vol. 14 | No. 3 |1551-1557| July - September | 2021

\section{Chemistry}

\section{RESULTS AND DISCUSSION}

We have prepared a series of 5-((9-ethyl-9H-carbazol-3-yl)methylene)thiazolidine-2,4-dione linked 1,2,3triazoles 3a-3kwhich was shown in Scheme-1.We have designed the synthesis in three steps. First,5-((9ethyl-9H-carbazol-3-yl)methylene)thiazolidine-2,4-dione(1) was prepared from 9-ethyl-9H-carbazole-3carbaldehyde and 2,4-thiazolidinedione using piperidine as a catalyst. Next, compound 1was propargyalted using potassium carbonate as a base in acetone solvent. Finally, the alkyne 2 was reacted with different azides in tetrahydrofuran solvent using copper iodide as a catalyst to get the 1,2,3-triazole.<smiles>CCn1c2ccccc2c2cc(C=O)ccc21</smiles><smiles>O=C1CSC(=O)N1</smiles>

Piperidene

Toluene, 4h, Reflux<smiles>CCn1c2ccccc2c2cc(/C=C3\SC(=O)NC3=O)ccc21</smiles>

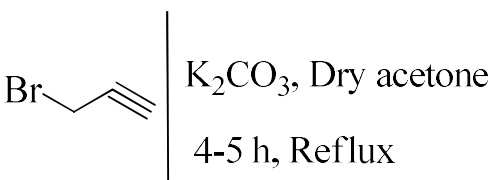<smiles>[R]c1ccc(-n2cc(CN3C(=O)S/C(=C\c4ccc5c(c4)c4ccccc4n5CC)C3=O)nn2)cc1</smiles>

3a-i

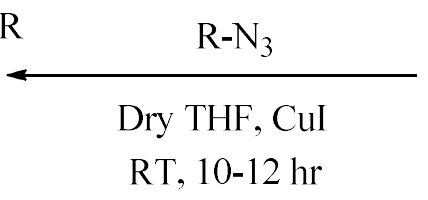

RT, 10-12 hr<smiles>C#CCN1C(=O)SC(=Cc2ccc3c(c2)c2ccccc2n3CC)C1=O</smiles>

$$
\begin{aligned}
& \mathrm{R}=\mathrm{H}(\mathbf{3 a}) ; \mathrm{R}=4-\mathrm{NO}_{2}(\mathbf{3 b}) ; \mathrm{R}=4-\mathrm{Ac}(\mathbf{3 c}) ; \mathrm{R}=3,4-\mathrm{Cl}(\mathbf{3 d}) ; \mathrm{R}=2-\mathrm{C}_{2} \mathrm{H}_{5}(\mathbf{3 e}) ; \\
& \mathrm{R}=4-\mathrm{F}(\mathbf{3} \mathbf{f}) ; \mathrm{R}=4-\mathrm{Cl}(\mathbf{3 g}) ; \mathrm{R}=4-\mathrm{Br}(\mathbf{3 h}) ; \mathrm{R}=4-\mathrm{OCH}_{3}(\mathbf{3 i})
\end{aligned}
$$

$3 \mathrm{j}$<smiles>CCCCCCN/C=C(/CN1C(=O)S/C(=C\c2ccc3c(c2)c2ccccc2n3CC)C1=O)N=N</smiles>

$3 \mathrm{k}$<smiles>CCCCCCCCNN1C=C(CN2C(=O)SC(=Cc3ccc4c(c3)c3ccccc3n4CC)C2=O)NN1</smiles>

Scheme-1: Synthesis of Thiazolidine-2,4-dionederivatives 3(a-k)

\section{In-vitro Anti-cancer Screening}

The newly prepared hybrids were screened for in vitro cytotoxic activity based on MTT assay against three human cancer lines MCF-7, HeLa and SKOV3. The various concentrations of the synthetic compounds (final concentration 1, 10, 20,30,40 and $50 \mu \mathrm{g} / \mathrm{ml}$ ) were applied to calculate $\mathrm{IC}_{50}$ and they are listed in Table-1. From Table-1 it is evident that compound 3b against MCF-7, compound 3i against HeLa and compound 3d against SKOV3, showed the highest activity with IC50 value of $32.92 \mu \mathrm{g} / \mathrm{ml}$, $12.08 \mu \mathrm{g} / \mathrm{ml}$ and $29.06 \mu \mathrm{g} / \mathrm{ml}$ respectively. Doxorubicin was used as a reference for in vitro anticancer screening.

Table-1: Anticancer Activity of Synthesized Compounds

\begin{tabular}{c|c|c|c}
\hline \multirow{2}{*}{ Compound } & \multicolumn{3}{|c}{ Anticancer Activity $\left(\mathrm{IC}_{50}\right.$ in $\left.\mu \mathrm{M}\right)$} \\
\cline { 2 - 4 } & MCF-7 & HeLa & SKOV3 \\
\hline $3 \mathrm{a}$ & 77.72 & 17.76 & 91.78 \\
\hline $3 \mathrm{~b}$ & 32.92 & 42.06 & $>100$ \\
\hline
\end{tabular}


RASĀYAN J. Chem. Vol. 14 | No. 3 |1551-1557| July - September | 2021

\begin{tabular}{c|c|c|c}
\hline $3 \mathrm{c}$ & $>100$ & 33.87 & $>100$ \\
\hline $3 \mathrm{~d}$ & $>100$ & 57.05 & 29.06 \\
\hline $3 \mathrm{e}$ & $>100$ & 32.36 & $>100$ \\
\hline $3 \mathrm{f}$ & $>100$ & 62.77 & $>100$ \\
\hline $3 \mathrm{~g}$ & 33.15 & 20.07 & $>100$ \\
\hline $3 \mathrm{~h}$ & $>100$ & 24.00 & $>100$ \\
\hline $3 \mathrm{i}$ & 91.66 & 12.08 & $>100$ \\
\hline $3 \mathrm{j}$ & $>100$ & 28.87 & 11.34 \\
\hline Doxorubicin & 35.29 & 02.96 &
\end{tabular}

\section{Biological Evaluation}

\section{Cytotoxicity Test (MTT Assay)}

The MTT assay was used for screening the in vitro anticancer activity of all the synthesized hybrids. Three different cancer cell lines SKOV3, MCF7 and HeLa were obtained from NCL Pune.These cell lines are maintained in Dulbecco's Modified Eagle's Medium where it is added with $10 \%$ Fetal bovine serum followed by $100 \mathrm{U} / \mathrm{ml}$ penicillin, $100 \mu \mathrm{g} / \mathrm{ml}$ streptomycin and $2 \mathrm{mM}$ 1-glutamine and human body temperature under $\mathrm{CO}_{2}$ incubator. Then the cells were seeded in 96 well culture plates. After adding the compounds in different concentrations ranging from 1 micromolar to 50 micromolar in triplicates were kept at room temperature for 24 hours. The cancer cells were incubated with MTT for $4 \mathrm{hr}$ and 100 microlitre dimethyl sulfoxide was added to each well and the absorbance was measured Synergy H1, the multi-mode plate reader. For this assay doxorubicin was used as a positive control.

\section{ACKNOWLEDGEMENT}

Auther ${ }^{1}$ is thankful to Sreenidhi Institute Science and Technology (SNIST), Hyderabad, Telangana, for providing lab facilities and K.L. Deemed to be University (KLES), Guntur, Andhra Pradesh for research support.

\section{CONCLUSION}

Here we demonstrated the synthesis and anti-cancer screening of 1,2,3-triazole linked thiazolidinecarbazole hybrids. The compounds $3 \mathrm{~b}, 3 \mathrm{i}$ and $3 \mathrm{~d}$ are effective in anticancer activity.

\section{REFERENCES}

1. M. Bernard, Accounts of Chemical Research, 41(1), 69(2008), https://doi.org/10.1021/ar7000843

2. H. Joshi, T. Pal and CS. Ramaa, Expert Opinion on Investigational Drugs, 23(4), 501(2014), https://doi.org/10.1517/13543784.2014.884708

3. F. Bray, J. Ferlay, I. Soerjomataram, R.L. Siegel, L.A. Torre and A. Jemal, CA: A Cancer Journal for Clinicians, 68, 394(2018), https://doi.org/10.3322/caac.21492

4. E. Fröhlich and R. Wahl, BioMed Research International, 2015, 1(2015), https://doi.org/10.1155/2015/845340

5. X. Jin, C.-J. Zheng, M.-X. Son, Y. Wu, L.-P. Sun, Y.-J. Li and H.R. Piao, European Journal of Medicinal Chemistry, 56, 203(2012), https://doi.org/10.1016/j.ejmech.2012.08.026

6. X.-F. Liu, C.-J. Zheng, L.-P. Sun, X.-K. Liu and H.-R. Piao, European Journal of Medicinal Chemistry, 46(8), 3469(2011), https://doi.org/10.1016/j.ejmech.2011.05.012

7. N. Sunduru, M. Sharma, K. Srivastava, S. Rajakumar, S.K. Puri, J.K. Saxena and P.M.S. Chauhan, Bioorganic \& Medicinal Chemistry, 17(17), 6451(2009), https://doi.org/10.1016/j.bmc.2009.05.075

8. L. Ma, C. Xie, Y. Ma, J. Liu, M. Xiang, X. Ye and L. Chen, Journal of Medicinal Chemistry, 54(7), 2060(2011), https://doi.org/10.1021/jm1011534

9. H. Chen, Y.-H. Fan, A. Natarajan, Y. Guo, J. Iyasere, F. Harbinski and J.A. Halperin, Bioorganic \& Medicinal Chemistry Letters, 14(21), 5401(2004), https://doi.org/10.1016/j.bmcl.2004.08.017

10. K. M. Pandya and P. S. Desai, Rasayan Journal of Chemistry, 13(2), 1054(2020), https://doi.org/10.31788/RJC.2020.1325628

11. A. K. Mohammed Iqbal, A.Y. Khan, M.B. Kalashetti, N.S. Belavagi, Y.-D. Gong and I.A.M. Khazi, $\begin{array}{llll}\text { European Journal of Medicinal Chemistry, 53, 308(2012), } & \end{array}$ https://doi.org/10.1016/j.ejmech.2012.04.015 
RASĀYAN J. Chem.

Vol. 14 | No. 3 |1551-1557| July - September | 2021

12. B.B. Lohray, V. Bhushan, B.P. Rao, G.R. Madhavan, N. Murali, K.N. Rao and S. Subramaniam, Journal of Medicinal Chemistry, 41(10), 1619(1998), https://doi.org/10.1021/jm970444e

13. S. Heng, W. Tieu, S. Hautmann, K. Kuan, D.S. Pedersen, M. Pietsch and A.D. Abell, Bioorganic \& Medicinal Chemistry, 19(24), 7453(2011), https://doi.org/10.1016/j.bmc.2011.10.042

14. Y. Wu, H.-H. Tai and H. Cho, Bioorganic \& Medicinal Chemistry, 18(4), 1428(2010), https://doi.org/10.1016/j.bmc.2010.01.016

15. B. Geetha, G. Swarnalatha and G.V. Subba Reddy, Rasayan Journal of Chemistry, 12(3), 1063(2019), https://doi.org/10.31788/RJC.2019.1235165

16. A.W. Schmidt, K.R. Reddy and H.-J. Knölker, Chemical Reviews, 112(6), 3193(2012), https://doi.org/10.1021/cr200447s

17. P.-Y. Wang, H.-S. Fang, W.-B. Shao, J. Zhou, Z. Chen, B.-A. Song and S. Yang, Bioorganic \& Medicinal Chemistry Letters, 27(18), 4294(2017), https://doi.org/10.1016/j.bmcl.2017.08.040

18. T. Surendiran, International Journal of PharmTech Research, 8, 183(2015).

19. P. Sathiyachandran, P. Manogaran, V.N. Nesterov, V.V. Padma and K.J. Rajendra Prasad, European Journal of Medicinal Chemistry, 150, 851(2018), https://doi.org/10.1016/j.ejmech.2018.03.056

20. G. Surineni, S.K. Marvadi, P. Yogeeswari, D. Sriram and S. Kantevari, Bioorganic \& Medicinal Chemistry Letters, 28(9), 1610(2018), https://doi.org/10.1016/j.bmcl.2018.03.048

21. B. Dinesh Kumar, M. Analava and M. Manjunatha, International Journal of Phytomedicine, 2, 22(2010), https://doi.org/10.5138/ijpm.2010.0975.0185.02004

22. Y. Hieda, M. Anraku, T. Choshi, H. Tomida, H. Fujioka, N. Hatae and S. Hibino, Bioorganic \& Medicinal Chemistry Letters, 24(15), 3530(2014), https://doi.org/10.1016/j.bmcl.2014.05.050

23. H. Yan, T.C. Mizutani, N. Nomura, T. Takakura, Y. Kitamura, H. Miura and W. Sugiura, Antiviral Chemistry and Chemotherapy, 16(6), 363(2005), https://doi.org/10.1177/095632020501600603

24. H. Jiang, W.-J. Zhang, P-H. Li, J. Wang, C.-Z. Dong, K. Zhang and Z.-Y. Du, Bioorganic \& Medicinal Chemistry Letters, 28(8), 1320(2018), https://doi.org/10.1016/j.bmcl.2018.03.017

25. S. Thiratmatrakul, C. Yenjai, P. Waiwut, O. Vajragupta, P. Reubroycharoen, M. Tohda and C. Boonyarat, European Journal of Medicinal Chemistry, 75, 21(2014), https://doi.org/10.1016/j.ejmech.2014.01.020

[RJC-6249/2020] 